ఠ

\title{
Profile of trifluridine/tipiracil hydrochloride in the treatment of metastatic colorectal cancer: efficacy, safety, and place in therapy
}

This article was published in the following Dove Press journal:

OncoTargets and Therapy

15 September 2017

Number of times this article has been viewed

\author{
Yu Sunakawa \\ Naoki Izawa \\ Takuro Mizukami \\ Yoshiki Horie \\ Mami Hirakawa \\ Hiroyuki Arai \\ Takashi Ogura \\ Takashi Tsuda \\ Takako Eguchi Nakajima \\ Department of Clinical Oncology, \\ St Marianna University School of \\ Medicine, Kawasaki, Japan
}

Correspondence: Yu Sunakawa Department of Clinical Oncology, St Marianna University School of Medicine, 2-I6-I, Sugao, Miyamae-ku, Kawasaki, Kanagawa 216-85I I, Japan Tel +8I 449778 I II

Fax +8I 449773835

Email y.suna0825@gmail.com
Abstract: TAS-102, with its robust survival efficacy and feasible toxicity, is one of the standard salvage-line treatments for patients with metastatic colorectal cancer (mCRC). No definitive data are available to determine which drug should be administered first during salvage-line treatment. Therefore, it is imperative that we establish the sequence of administration by considering drug toxicity profiles based on patient characteristics, such as age, performance status, comorbidities, tolerability to previous treatments, and patient preferences. The identification of predictive biomarkers in response to TAS-102 or its toxicity is urgently needed for better patient selection. Moreover, to strengthen efficacy or relieve toxicity, combinations with other agents, which could potentially emerge as standard treatment regimens, have been investigated and compared to existing active regimens for mCRC.

Keywords: TAS-102, metastatic colorectal cancer, regorafenib, biomarker

\section{Introduction}

Colorectal cancer (CRC) is the third most common cancer worldwide. ${ }^{1}$ More than $20 \%$ of patients with CRC are initially diagnosed with metastatic disease, while more than one-third of those with stage III CRC eventually experience recurrence after curative surgery. The 5-year survival rate of patients with metastatic colorectal cancer (mCRC) is particularly low at $13 \% .^{2}$ More effective or intensive chemotherapy may provide an opportunity for secondary resection of metastatic disease, which could potentially lead to improved survival and cure. ${ }^{3,4}$ However, given the difficulty in the treatment of advanced diseases, a number of patients with $\mathrm{mCRC}$ receive palliative chemotherapy to prolong survival time.

TAS-102 is an orally administered combination of trifluridine (FTD), a thymidinebased nucleoside analog, and tipiracil hydrochloride, a thymidine phosphorylase inhibitor (TPI). TAS-102 was initially approved in Japan in March 2014 and was subsequently approved by the US Food and Drug Administration in September 2015.5 It was then approved in the European Union in April 2016 after an international phase III clinical trial demonstrated clear survival benefits compared to placebo. ${ }^{6}$

In the present review, we summarize the development of TAS-102 from the first pilot study, as well as recent findings derived from several clinical studies. In particular, we focus on the role of TAS-102 in the treatment of $\mathrm{mCRC}$, treatment selection based on patient characteristics, such as age, performance status, and comorbidities, and possible predictive biomarkers to refine the salvage-line clinical management of patients with mCRC. 


\section{The beginning of the story:TAS- 102 development}

The primary cytotoxic mechanism of oral FTD is DNA dysfunction through the incorporation of its phosphate form into the DNA by thymidine kinase. ${ }^{7,8}$ This mechanism is distinct from those of 5-FU and other fluoropyrimidines, which derive their cytotoxic effects from the inhibition of thymidylate synthase. Moreover, TPI improves the bioavailability of FTD by inhibiting its catabolism by thymidine phosphorylase. ${ }^{9}$ The combination of these two agents has made TAS-102 as one of the new treatments beneficial for patients with mCRC who are refractory to fluoropyrimidines.

The schedule of TAS-102 administration had been investigated in several Phase I trials, as well as in Japanese Phase I and II trials, and was subsequently confirmed in a more recent Phase I trial evaluating the safety of TAS-102 in the United States (US). ${ }^{10}$ The first Phase I trial was conducted in 14 patients. According to preclinical studies, TAS-102 was given orally once daily for 14 days with a 1 -week rest period, which was repeated every 3 weeks. The maximum tolerated dose was found to be $50 \mathrm{mg} / \mathrm{m}^{2} /$ day, whereas the dose-limiting toxicity was neutropenia at a dose of $60 \mathrm{mg} / \mathrm{m}^{2} /$ day. ${ }^{11}$ Additional Phase I trials were designed to determine the best TAS-102 schedule that minimizes hematological toxicity. These Phase I trials evaluated two TAS-102 schedules: once daily on days $1-5$ and $8-12$ every 28 days or on days $1-5$ every 21 days. The recommended doses (RDs) were 100 and $160 \mathrm{mg} / \mathrm{m}^{2} /$ day on the first and second regimens, respectively. The doses (100 and $160 \mathrm{mg} / \mathrm{m}^{2} /$ day) were subsequently evaluated in 12 patients with solid tumors resistant to $5-\mathrm{FU}$, though no objective responses were observed. ${ }^{12}$ Thereafter, Phase I trials that administered TAS-102 on days 1-5 and 8-12 every 4 weeks were performed to compare thrice- and twice-a-day administration. The Phase I trial that administered TAS-102 three times a day to their patients, $85 \%$ of which had CRC, determined a RD of $70 \mathrm{mg} / \mathrm{m}^{2} /$ day and showed stable disease in 9 out of 15 patients. ${ }^{13}$ Based on the aforementioned Phase I trials and considering efficacy and toxicity, a RD of $50 \mathrm{mg} / \mathrm{m}^{2}$ twice a day, 5 days a week for 2 weeks, was established in the US. A Phase I trial in Japan that administered TAS-102 twice a day on days $1-5$ and days 8-12 for 4 weeks was conducted in 21 patients with solid tumors that were refractory to standard chemotherapy to determine the optimal Phase II dose and examine the pharmacokinetic (PK) profile. PK data indicated that systemic concentrations of FTD and TPI increased linearly with increasing oral doses, while dose-dependent increases were observed for doses up to $70 \mathrm{mg} / \mathrm{m}^{2} /$ day. Therefore, a RD of $70 \mathrm{mg} / \mathrm{m}^{2} /$ day was determined for future Phase II trials. The disease control rate (DCR) was $50 \%$ with a median progression-free survival (PFS) of 2.4 months in 18 patients with mCRC..$^{14}$ Hence, the schedule and dosage of TAS-102 administration were reconfirmed in another US Phase I trial. ${ }^{10}$

To investigate the efficacy and safety of TAS-102, a multicenter, double-blind, randomized Phase II trial was conducted in Japan involving patients with mCRC refractory to 5-FU, irinotecan, and oxaliplatin. Patients were randomized in a 2:1 ratio to either TAS-102 plus best supportive care (BSC) or placebo plus BSC. The primary endpoint of this study was overall survival (OS). The TAS-102 group had a better median survival time compared to the placebo group (9.0 months vs 6.6 months; hazard ratio [HR] in OS 0.56; $95 \%$ confidence interval $[\mathrm{CI}] 0.39-0.81 ; P=0.001) .{ }^{15}$

\section{Effectiveness across the world}

A significant result regarding survival benefit from the Japanese Phase II trial showed that the efficacy of TAS-102 was assessed across the world through the Phase III RECOURSE trial. A total of 800 patients with $\mathrm{mCRC}$ refractory or intolerant to 5-FU, irinotecan, oxaliplatin, bevacizumab, and anti-epidermal growth factor receptor (EGFR) antibodies of wild-type $K R A S$ were randomly assigned in a $2: 1$ ratio to either TAS-102 $\left(35 \mathrm{mg} / \mathrm{m}^{2}\right.$ per dose twice daily on days $1-5$ and 8-12, every 4 weeks) or placebo in Japan, US, Europe, and Australia. Regorafenib became available for the management of previously treated colorectal cancer during the course of the study. The primary endpoint was OS, whereas PFS, response rate, DCR, and safety were included as secondary endpoints. Approximately $60 \%$ of the patients in both treatment arms had previously received four or more treatment regimens. The results demonstrated that compared to placebo, the TAS-102 group had significantly better OS (HR 0.68; 95\% CI 0.58-0.81; $P<0.001$; median OS: 7.1 months vs 5.3 months) and PFS (HR $0.48 ; 95 \%$ CI $0.41-0.57 ; P<0.001$; median PFS: 2.0 months vs 1.7 months). DCR was also significantly better in the TAS-102 group than in the placebo group $(44.0 \%$ vs $16.3 \% ; P<0.0001) .{ }^{6}$

The RECOURSE Spanish subgroup reported significant improvements in OS and PFS with TAS-102 versus placebo, which was consistent with the overall RECOURSE population. No new safety signals were identified..$^{16}$ This subgroup analysis indicates that European and Japanese patients have comparable TAS-102 activity.

\section{Practical issues: toxicities}

In the previous Phase II trial and RECOURSE trial, neutropenia was the most common adverse event in patients treated 
with TAS-102. This required at least one dose reduction and/ or treatment interruption in up to a third of the patients. Other common adverse events of TAS-102 included leukopenia, lymphocytopenia, and anemia for hematologic toxicity and nausea, appetite loss, fatigue, and diarrhea for non-hematologic toxicity. $6,11,14,15$ TAS-102 was generally well tolerated according to a safety report from the RECOURSE trial, while no new safety concerns were observed in the European subgroup during the Phase III trial. Neutropenia was the most frequent and clinically significant adverse event in the European subgroup. The only substantial difference in the safety profile of TAS-102 was that European patients had higher incidences of asthenia and lower incidences of fatigue compared to the overall RECOURSE trial population. The frequency of serious adverse events and hospitalizations was somewhat lower in the European population than in the overall population. However, this may have been due to local differences in healthcare expenditure, socioeconomic status, and lifestyle, ${ }^{17}$ as well as the relatively small number of patients in this population.

Reports have shown that TAS-102 has been consistently safe in clinical practice regardless of prior regorafenib use. Retrospective studies that have investigated safety profiles in patients receiving both TAS-102 and regorafenib demonstrated comparable toxicity between those receiving TAS-102 before and after regorafenib. However, patients who received regorafenib after TAS-102 tended to have higher rates of grade 3 or 4 anemia, while those who received TAS-102 after regorafenib tended to have higher rates of grade 3 or 4 leukopenia and neutropenia. ${ }^{18,19}$ The safety profile of TAS-102 in patients with poor Eastern Cooperative Oncology Group (ECOG) performance status (PS) remains unclear, because patients with ECOG PS 2 were not enrolled in the RECOURSE trial.

\section{The role of TAS-I02 in the treatment of $\mathrm{mCRC}$}

TAS-102 is recognized as a standard salvage-line treatment for patients previously treated with 5-FU, irinotecan, and oxaliplatin plus targeted agents in the National Comprehensive Cancer Network guideline, which is similar to the Japanese CRC treatment guideline. ${ }^{20}$ Both TAS-102 and regorafenib serve as key chemotherapeutic agents in salvage treatment, because Phase III clinical trials for each drug showed similar survival benefits compared to placebo. However, both drugs had very different safety profiles due to completely different mechanisms of action. Regorafenib has been associated with fatigue, hand-foot skin reactions, diarrhea, voice changes, hypertension, rashes, etc., while
Table I Comparison of Phase III trials evaluating TAS-I02 and regorafenib

\begin{tabular}{|c|c|c|}
\hline \multirow[t]{2}{*}{ Trial } & \multirow{2}{*}{$\begin{array}{l}\text { RECOURSE } \\
\text { TAS-I } 02 \text { vs } \\
\text { placebo }\end{array}$} & \multirow{2}{*}{$\begin{array}{l}\text { CORRECT } \\
\text { Regorafenib vs } \\
\text { placebo }\end{array}$} \\
\hline & & \\
\hline $\mathrm{N}$ & 534 vs 266 & 505 vs 255 \\
\hline Median PFS (months) & 2.0 vs 1.7 & 1.9 vs 1.7 \\
\hline HR $(95 \% \mathrm{Cl})$ & $0.48(0.4 \mathrm{I}-0.57)$ & $0.49(0.42-0.58)$ \\
\hline Median OS (months) & 7.1 vs 5.3 & 6.4 vs 5.0 \\
\hline HR $(95 \% \mathrm{Cl})$ & $0.68(0.58-0.8 \mathrm{I})$ & $0.77(0.64-0.94)$ \\
\hline Any grade AEs (\%) & 98 vs 93 & 93 vs 61 \\
\hline Grade 3-4 AEs (\%) & 69 vs 52 & 54 vs 14 \\
\hline Most relevant AEs & $\begin{array}{l}\text { Neutropenia, anemia, } \\
\text { nausea, appetite loss }\end{array}$ & $\begin{array}{l}\text { Hand-foot skin reaction, } \\
\text { rash, fatigue, hypertension }\end{array}$ \\
\hline
\end{tabular}

Abbreviations: PFS, progression-free survival; $\mathrm{HR}$, hazard ratio; $\mathrm{Cl}$, confidence interval; OS, overall survival; AEs, adverse events.

TAS-102 has been associated with nausea, vomiting, fatigue, diarrhea, neutropenia etc. (Table 1). ${ }^{6,21}$

Retrospective studies have shown that TAS-102 and regorafenib have not only different toxicity profiles but also comparable efficacy in patients with $\mathrm{mCRC}$. One retrospective study reported that patients $(\mathrm{n}=6)$ who received crossover treatment from regorafenib to TAS-102 had a median PFS and OS of 4.7 and 11.5 months, respectively, while those $(n=8)$ who received crossover treatment from TAS-102 to regorafenib had a median PFS and OS of 3.7 and 7.6 months, respectively, ${ }^{22}$ suggesting that the order of the salvageline regimens may be associated with survival. However, a sub-analysis of another retrospective study using a bigger cohort showed no significant difference in OS between both groups $(P=0.99)$. This study indicated no statistically significant difference in PFS between the regorafenib and TAS-102 groups (median PFS: 2.1 months vs 2.1 months) after univariate (HR, $1.20 ; 95 \% \mathrm{CI}, 0.87-1.67 ; P=0.27$ ) and multivariate (adjusted HR, 1.27; 95\% CI, 0.90-1.83; $P=0.18$ ) analyses. The median OS was 6.7 and 6.5 months in the regorafenib and TAS-102 groups, respectively. No statistically significant difference in OS was found after univariate (HR, 1.01; 95\% CI, 0.70-1.49; $P=0.97$ ) or multivariate (adjusted HR, 0.98; 95\% CI, 0.65-1.51; $P=0.92$ ) analysis. The number of dose modifications and interruptions due to adverse events was significantly greater in the regorafenib group than in the TAS-102 group. The median time to treatment failure (TTF) was 1.8 and 2.0 months in the regorafenib and TAS-102 groups (HR, 1.22; 95\% CI, $0.89-1.70 ; P=0.21)$, respectively. Interestingly, subgroup analysis showed that elderly individuals with poor ECOG PS and higher lactate dehydrogenase levels in the regorafenib group tended to have shorter PFS than those in the TAS-102 
group. ${ }^{19}$ In addition, another retrospective study showed that TAS-102 and regorafenib had comparable PFS and OS despite prior regorafenib use. ${ }^{18}$

The REGOTAS trial, another retrospective study, has recently reported clinical data on patients who were treated with TAS-102 and/or regorafenib collected from 24 institutions of the Japanese Society for Cancer of the Colon and Rectum. Clinical outcomes in 550 patients (223 and 327 in the regorafenib and TAS-102 groups, respectively) were evaluated using the Kaplan-Meier method and Cox models based on propensity score adjustment and matching for OS as the primary endpoint. OS was similar between both groups (HR of TAS-102 to regorafenib, 0.96; 95\% CI, 0.78-1.18). No statistically significant differences between both groups were found for PFS (HR 0.94) and time to ECOG PS $\geq 2$ deterioration (HR 1.00). However, TTF was significantly longer in the TAS-102 group compared to the regorafenib group (HR $0.81 ; P=0.025$ ). In addition, exploratory subanalysis was performed to investigate the impact of age on survival. The results indicated that the regorafenib group had favorable survival among those aged $<65$ years but unfavorable survival among those aged $\geq 65$ years compared to the TAS-102 group $(P$ for interaction $=0.012)($ Table 2$) .{ }^{23}$
Overall, no definitive data are available to determine which drug should be administered first during salvage-line treatment. Therefore, it is imperative that we establish the sequence of administration by considering drug toxicity profiles based on patient characteristics, such as age, PS, comorbidities, tolerability to previous treatments, and patient preferences. Maximizing the availability of both drugs may be a clinically important treatment strategy to prolong survival during poor prognostic conditions.

\section{Promising predictive biomarkers}

Although data regarding molecular pathways related to CRC have been accumulated, few clinically useful prognostic markers for CRC-specific survival have been recognized. BRAF V600E mutation in mCRC is the only notable exception. ${ }^{24}$ Moreover, only limited data are available concerning predictive markers for any specific agents, with the only validated marker being $R A S$ mutations, a well-known predictive marker for resistance to anti-EGFR antibodies. ${ }^{25,26}$

Sub-analyses of previous trials were performed to investigate the impact of KRAS and BRAF status on TAS-102 efficacy. The efficacy of TAS-102 was similar in all patients irrespective of their KRAS mutation status. ${ }^{6}$ Nevertheless,

Table 2 Retrospective studies evaluating TAS-102 and regorafenib as salvage-line treatment

\begin{tabular}{|c|c|c|c|c|c|c|}
\hline \multirow{2}{*}{$\begin{array}{l}\text { Study } \\
\text { Treatment }\end{array}$} & \multicolumn{2}{|c|}{ Masuishi et al ${ }^{19}$} & \multicolumn{2}{|c|}{ Sueda et $\mathrm{al}^{22}$} & \multicolumn{2}{|c|}{ REGOTAS $^{23}$} \\
\hline & TAS- 102 & Regorafenib & TAS- 102 & Regorafenib & TAS- 102 & Regorafenib \\
\hline $\mathrm{N}$ & 50 & 146 & 14 & 23 & 327 & 223 \\
\hline Response rate & $0 \%$ & $0.8 \%$ & $0 \%$ & $0 \%$ & & \\
\hline \multirow[t]{2}{*}{ Disease control rate } & $38 \%$ & $33 \%$ & $29 \%$ & $30 \%$ & & \\
\hline & \multicolumn{2}{|c|}{$P=0.60$} & & & & \\
\hline \multirow[t]{3}{*}{ Median PFS } & $\begin{array}{l}2.1 \text { months } \\
95 \% \mathrm{Cl} \mathrm{I.8-3.1}\end{array}$ & $\begin{array}{l}2.1 \text { months } \\
95 \% \mathrm{Cl} 1.8-2.5\end{array}$ & $\begin{array}{l}2.1 \text { months } \\
95 \% \mathrm{Cl} 0.92-6.39\end{array}$ & $\begin{array}{l}3.0 \text { months } \\
95 \% \mathrm{Cl} \mathrm{I.64-4.52}\end{array}$ & $\begin{array}{l}2.1 \text { months } \\
95 \% \mathrm{Cl} 2.0-2.3\end{array}$ & $\begin{array}{l}2.1 \text { months } \\
95 \% \mathrm{Cl} 2.0-2.4\end{array}$ \\
\hline & \multicolumn{2}{|c|}{ HR I.20 95\% Cl 0.87-I.67 P=0.27 } & \multicolumn{2}{|l|}{$P=0.59$} & & \\
\hline & \multicolumn{2}{|c|}{$\begin{array}{l}\text { Adjusted HR I.27 } \\
95 \% \mathrm{Cl} 0.90-1.83 P=0.18\end{array}$} & & & \multicolumn{2}{|c|}{$\begin{array}{l}\text { Adjusted HR } 0.94 \\
P=0.54\end{array}$} \\
\hline \multirow[t]{3}{*}{ Median OS } & $\begin{array}{l}6.5 \text { months } \\
95 \% \mathrm{Cl} 5.3-8.3\end{array}$ & $\begin{array}{l}6.7 \text { months } \\
95 \% \mathrm{Cl} 5.8-7.6\end{array}$ & $\begin{array}{l}6.3 \text { months } \\
95 \% \mathrm{Cl} 3.21-9.93\end{array}$ & $\begin{array}{l}5.8 \text { months } \\
95 \% \mathrm{Cl} 3.7-1 \mathrm{I} .7\end{array}$ & $\begin{array}{l}7.4 \text { months } \\
95 \% \mathrm{Cl} 6.8-8.3\end{array}$ & $\begin{array}{l}7.9 \text { months } \\
95 \% \mathrm{Cl} 6.8-9.2\end{array}$ \\
\hline & \multicolumn{2}{|c|}{$\begin{array}{l}\mathrm{HR} \text { I.0I } 95 \% \mathrm{Cl} 0.70-1.49 \\
P=0.97\end{array}$} & \multicolumn{2}{|l|}{$P=0.60$} & & \\
\hline & \multicolumn{2}{|c|}{$\begin{array}{l}\text { Adjusted HR } 0.98 \\
95 \% \mathrm{Cl} 0.65-1.5 \mid P=0.92\end{array}$} & & & \multicolumn{2}{|c|}{$\begin{array}{l}\text { Adjusted HR } 0.96 \\
95 \% \mathrm{Cl} 0.78-1.18 P=0.69\end{array}$} \\
\hline Subsequent chemotherapy & $44 \%$ & $54 \%$ & & & $50 \%$ & $65 \%$ \\
\hline TAS- 102 & $0 \%$ & $30 \%$ & $0 \%$ & $26 \%$ & $0 \%$ & $59 \%$ \\
\hline Regorafenib & $28 \%$ & $0 \%$ & $57 \%$ & $0 \%$ & $37 \%$ & $0 \%$ \\
\hline \multicolumn{7}{|c|}{ Reason for treatment discontinuation } \\
\hline Disease progression & $94 \%$ & $86 \%$ & $86 \%$ & $61 \%$ & $92 \%$ & $75 \%$ \\
\hline Adverse events & $6 \%$ & $12 \%$ & $14 \%$ & $26 \%$ & $7 \%$ & $24 \%$ \\
\hline
\end{tabular}

Abbreviations: PFS, progression-free survival; $\mathrm{HR}$, hazard ratio; OS, overall survival; $\mathrm{Cl}$, confidence interval. 
one clinical trial showed that TAS-102 seemed to have more pronounced OS and PFS benefits in patients with KRAS mutations, for which no reasonable biological explanation has been adapted so far. ${ }^{15}$ Moreover, although no differences in OS and PFS were observed between wild-type $B R A F$ and $B R A F$-mutated tumors, no definitive conclusions can be made due to the small sample size.

A retrospective study including 181 patients treated with TAS-102 investigated whether genetic polymorphisms in homologous recombination and cell-cycle checkpoint pathways for DNA repair were associated with clinical outcomes. ATM gene polymorphisms were identified as a candidate predictor of TAS-102 efficacy. Patients with any $\mathrm{G}$ allele in $A T M$ rs609429 had better OS than those with the $\mathrm{C} / \mathrm{C}$ variant (median: 8.7 months vs 4.4 months, HR $0.37, P=0.022$ ), which remained significant even after multivariable analysis. ${ }^{27} \mathrm{~A}$ retrospective study of two randomized Phase II and III trials was performed to assess the association between thymidine kinase 1 (TK1) expression, which induces incorporation of FTD into DNA, and TAS-102 efficacy. The study indicated that among patients with high TK1 expression, those receiving placebo had poor prognosis, while those receiving TAS-102 showed significant improvements in OS. ${ }^{28}$ Although these findings may show that TK1 expression could be a potential predictive marker for TAS-102, further validation is still needed.

On the other hand, clinical markers to predict outcomes of TAS-102 treatment have also been investigated. Kasi et al conducted a cohort study in which chemotherapy-induced neutropenia (CIN) 1 month after starting TAS-102 appeared to be a prognostic and/or predictive factor of clinical outcomes. In their study, CIN was significantly associated with better prognosis in patients treated with TAS-102. Those who experienced $\geq$ grade $2 \mathrm{CIN}$ after 1 month had longer PFS (median 3.0 months vs 2.4 months; $P=0.01$ ) and OS (median 14.0 months vs 5.6 months; $P<0.0001$ ). Moreover, OS remained statistically significant even after multivariate analysis (adjusted HR 0.21; 95\% CI 0.11-0.38). These findings have led us to hypothesize that an increase in the TAS-102 dosage may be needed to achieve better outcomes in patients not experiencing any neutropenia. ${ }^{29}$ Predictive and/or prognostic markers for TAS-102 in combination with other cytotoxic and/or biologic drugs have still been under investigation.

In summary, valid predictors for TAS-102 efficacy have yet to be discovered. The detection of such biomarkers may improve the selection of patients who can benefit from
TAS-102 and consequently the best active drug for patients with refractory $\mathrm{mCRC}$, which prevents unnecessary toxicities and optimizes the treatment strategy.

\section{Future perspectives on the TAS-102 regimen}

The evaluation of TAS-102 in combination with other active drugs, such as irinotecan, oxaliplatin, antiangiogenetics, and anti-EGFR antibodies, for $\mathrm{mCRC}$ may be promising. Temmink et al have shown that FTD potentiates oxaliplatininduced adduct formation, leading to a synergistic effect in colon cancer cell lines. ${ }^{30} \mathrm{SN} 38$-induced cytotoxicity in human colon cancer cell lines was increased after pre-incubation with FTD. ${ }^{31}$ These in vitro preclinical findings were later confirmed in vivo using colorectal xenograft nude mouse models, because the combination of TAS-102 with oxaliplatin ${ }^{32}$ or irinotecan ${ }^{33}$ produced higher growth inhibitory activity. Combinations of TAS-102 with bevacizumab or anti-EGFR antibodies were also investigated in the preclinical setting, showing significantly better effectiveness in tumor regression of CRC xenografts than TAS-102 monotherapy. ${ }^{34}$ A preclinical study provided a compelling rationale for evaluating the combination of anti-EGFR antibodies and TAS-102 for mCRC. Both colon cancer cells and colon cancer patient-derived xenograft models showed that panitumumab in combination with TAS-102 leads to additive and synergistic antiproliferative effects via blocking of EGFRmediated tumor response to FTD. ${ }^{35}$ In addition, the antitumor effects of TAS-102 plus anti-PD-1 antibody were studied in a syngeneic mouse colorectal cancer model. The combination of TAS-102 and anti-PD-1 antibody inhibited in vivo tumor growth at significantly higher rates than monotherapy. Flow cytometry analysis revealed that the combination increased $\mathrm{CD}^{+} \mathrm{T}$-cell percentages in whole lymphocyte and decreased regulatory $\mathrm{T}$-cell percentages in $\mathrm{CD}^{+}{ }^{+} \mathrm{T}$ cells. ${ }^{36}$

A Japanese Phase I trial evaluating the combination of TAS-102 and irinotecan was recently reported. A total of 10 patients were enrolled, and the recommended TAS-102 and irinotecan doses were 50 and $150 \mathrm{mg} / \mathrm{m}^{2}$, respectively. ${ }^{37}$ C-TASK FORCE, a Phase I/II trial, was performed to evaluate the efficacy and safety of TAS-102 with bevacizumab in 25 patients with $\mathrm{mCRC}$ refractory to standard therapies. The report showed that the trial met its primary endpoint, a PFS rate of $42.9 \%$ at 16 weeks. The combination of TAS-102 and bevacizumab showed promising antitumor activity with acceptable toxicity, with median PFS and OS of 5.6 and 11.2 months, respectively, as assessed by the investigators. ${ }^{38}$ Given that no consensus is available on which administration 
Table 3 Ongoing clinical trials evaluating TAS- 102 regimens

\begin{tabular}{|c|c|c|c|}
\hline Trial $^{\mathrm{a}}$ & Phase & Trial ID & Treatment \\
\hline \multicolumn{4}{|l|}{ First line } \\
\hline ALEXANDRIA & II & NCT02654639 & TAS-I02 with bevacizumab maintenance therapy post-induction chemo \\
\hline TASCOI & II & NCT0274322I & $\begin{array}{l}\text { TAS- } 102 \text { plus bevacizumab vs capecitabine plus bevacizumab for patients non-eligible for } \\
\text { intensive chemo }\end{array}$ \\
\hline KSCCI602 & I & UMIN00002524I & TAS- I02 plus bevacizumab for the elderly \\
\hline \multicolumn{4}{|l|}{ Second line } \\
\hline TRUSTY & II & JapicCTI-|736/8 & TAS plus bevacizumab vs FOLFIRI plus bevacizumab \\
\hline T-CORE & II & UMIN0000I7589 & TAS- 102 alone for the elderly \\
\hline MODURATE & 1 & UMIN0000I9828 & TAS-I02 plus irinotecan with bevacizumab \\
\hline CLI-95005-00I & I & NCT02848443 & TAS- 102 plus oxaliplatin ( \pm bevacizumab or nivolumab) \\
\hline TAS-102-109 & I & NCT01916447 & TAS-I02 plus irinotecan with bevacizumab \\
\hline \multicolumn{4}{|l|}{ Salvage line } \\
\hline WJOG8916G & II & UMIN000027210 & TAS-I02 plus cetuximab for RAS wild-type patients refractory to previous anti-EGFR antibodies \\
\hline TAS-102-203 study & I & NCT02860546 & TAS-I02 plus nivolumab for patients with MSS CRC \\
\hline TAS-OX & $\mathrm{I} / \mathrm{II}$ & NCT02848079 & TAS- 102 plus oxaliplatin \\
\hline APOLLON & $\mathrm{I} / \mathrm{II}$ & NCT026I322I & TAS-I02 plus panitumumab \\
\hline N-TASK FORCE & $\mathrm{I} / \mathrm{II}$ & UMIN0000I7II4 & TAS- I02 plus nintedanib \\
\hline
\end{tabular}

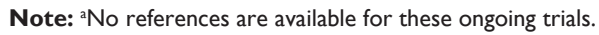

Abbreviations: FOLFIRI, fluorouracil/levofolinate calcium plus irinotecan; EGFR, epidermal growth factor receptor; MSS, microsatellite stable; CRC, colorectal cancer.

sequence of TAS-102 and regorafenib is more beneficial in salvage-line treatment of refractory $\mathrm{mCRC}$, the combination of TAS-102 and bevacizumab could potentially be a good option in current clinical practice. The use of TAS-102 for not only up-front but also maintenance therapy has been evaluated in clinical trials. Moreover, many clinical trials that assess the efficacy of combination strategies with TAS-102 are ongoing (Table 3 ).

\section{Conclusion}

TAS-102, with its robust survival efficacy and feasible toxicity, is one of the standard salvage-line treatments for patients with mCRC. The identification of predictive biomarkers in response to TAS-102 or its toxicity is urgently needed for better patient selection. Moreover, to strengthen efficacy or relieve toxicity, combinations with other agents, which could potentially emerge as standard treatment strategies, have been investigated and compared to existing active regimens for mCRC. Combinations with immuno-oncology agents have also been investigated for enriched patients, which may generate novel biological strategies for the treatment of mCRC.

\section{Disclosure}

Author YS has received honoraria from Taiho Pharmaceutical and Bayer. The other authors report no conflicts of interest in this work.

\section{References}

1. Ferlay J, Shin HR, Bray F, Forman D, Mathers C, Parkin DM. Estimates of worldwide burden of cancer in 2008: GLOBOCAN 2008. Int J Cancer. 2010;127(12):2893-2917.
2. Siegel R, Ma J, Zou Z, Jemal A. Cancer statistics, 2014. CA Cancer J Clin. 2014;64(1):9-29.

3. Nordlinger B, Van Cutsem E, Gruenberger T, et al; European Colorectal Metastases Treatment Group; Sixth International Colorectal Liver Metastases Workshop. Combination of surgery and chemotherapy and the role of targeted agents in the treatment of patients with colorectal liver metastases: recommendations from an expert panel. Ann Oncol. 2009;20(6):985-992.

4. Folprecht G, Gruenberger T, Bechstein WO, et al. Tumour response and secondary resectability of colorectal liver metastases following neoadjuvant chemotherapy with cetuximab: the CELIM randomised phase 2 trial. Lancet Oncol. 2010;11(1):38-47.

5. Marcus L, Lemery SJ, Khasar S, et al. FDA approval summary: TAS-102. Clin Cancer Res. 2017;23(12):2924-2927.

6. Mayer RJ, Van Cutsem E, Falcone A, et al; RECOURSE Study Group. Randomized trial of TAS-102 for refractory metastatic colorectal cancer. N Engl J Med. 2015;372(20):1909-1919.

7. Emura T, Murakami Y, Nakagawa F, Fukushima M, Kitazato K. A novel antimetabolite, TAS-102 retains its effect on FU-related resistant cancer cells. Int J Mol Med. 2004;13(4):545-549.

8. Tanaka N, Sakamoto K, Okabe H, et al. Repeated oral dosing of TAS102 confers high trifluridine incorporation into DNA and sustained antitumor activity in mouse models. Oncol Rep. 2014;32(6):2319-2326.

9. Emura T, Suzuki N, Fujioka A, Ohshimo H, Fukushima M. Potentiation of the antitumor activity of alpha, alpha, alpha-trifluorothymidine by the co-administration of an inhibitor of thymidine phosphorylase at a suitable molar ratio in vivo. Int J Oncol. 2005;27(2):449-455.

10. Bendell JC, Rosen LS, Mayer RJ, et al. Phase 1 study of oral TAS-102 in patients with refractory metastatic colorectal cancer. Cancer Chemother Pharmacol. 2015;76(5):925-932.

11. Hong DS, Abbruzzese JL, Bogaard K, et al. Phase I study to determine the safety and pharmacokinetics of oral administration of TAS-102 in patients with solid tumors. Cancer. 2006;107(6):1383-1390.

12. Overman MJ, Varadhachary G, Kopetz S, et al. Phase 1 study of TAS-102 administered once daily on a 5-day-per-week schedule in patients with solid tumors. Invest New Drugs. 2008;26(5):445-454.

13. Overman MJ, Kopetz S, Varadhachary G, et al. Phase I clinical study of three times a day oral administration of TAS-102 in patients with solid tumors. Cancer Invest. 2008;26(8):794-799.

14. Doi T, Ohtsu A, Yoshino T, et al. Phase I study of TAS-102 treatment in Japanese patients with advanced solid tumours. Br J Cancer. 2012; 107(3):429-434. 
15. Yoshino T, Mizunuma N, Yamazaki K, et al. TAS-102 monotherapy for pretreated metastatic colorectal cancer: a double-blind, randomised, placebo-controlled phase 2 trial. Lancet Oncol. 2012;13(10): 993-1001.

16. Longo-Muñoz F, Argiles G, Tabernero J, et al. Efficacy of trifluridine and tipiracil (TAS-102) versus placebo, with supportive care, in a randomized, controlled trial of patients with metastatic colorectal cancer from Spain: results of a subgroup analysis of the phase 3 RECOURSE trial. Clin Transl Oncol. 2017;19(2):227-235.

17. De Angelis R, Sant M, Coleman MP, et al; EUROCARE-5 Working Group. Cancer survival in Europe 1999-2007 by country and age: results of EUROCARE - 5-a population-based study. Lancet Oncol. 2014; 15(1):23-34

18. Kotani D, Shitara K, Kawazoe A, et al. Safety and efficacy of trifluridine/ tipiracil monotherapy in clinical practice for patients with metastatic colorectal cancer: experience at a single institution. Clin Colorectal Cancer. 2016;15(3):e109-e115.

19. Masuishi T, Taniguchi H, Hamauchi S, et al. Regorafenib versus trifluridine/tipiracil for refractory metastatic colorectal cancer: a retrospective comparison. Clin Colorectal Cancer. 2017;16(2):e15-e22.

20. Watanabe T, Muro K, Ajioka Y, et al. Japanese Society for Cancer of the Colon and Rectum (JSCCR) guidelines 2016 for the treatment of colorectal cancer. Int J Clin Oncol. Epub 2017 Mar 27.

21. Grothey A, Van Cutsem E, Sobrero A, et al; CORRECT Study Group. Regorafenib monotherapy for previously treated metastatic colorectal cancer (CORRECT): an international, multicentre, randomised, placebo-controlled, phase 3 trial. Lancet. 2013;381(9863):303-312.

22. Sueda T, Sakai D, Kudo T, et al. Efficacy and safety of regorafenib or TAS-102 in patients with metastatic colorectal cancer refractory to standard therapies. Anticancer Res. 2016;36(8):4299-4306.

23. Fukuoka S, Moriwaki T, Taniguchi H, et al. Regorafenib (REG) versus trifluridine/tipiracil (TAS-102) as salvage-line in patients with metastatic colorectal cancer refractory to standard chemotherapies (REGOTAS): a propensity score analysis from a JSCCR multicenter observational study. J Clin Oncol. 2017;35(15 Suppl):3540.

24. Tran B, Kopetz S, Tie J, et al. Impact of BRAF mutation and microsatellite instability on the pattern of metastatic spread and prognosis in metastatic colorectal cancer. Cancer. 2011;117(20):4623-4632.

25. Douillard JY, Oliner KS, Siena S, et al. Panitumumab-FOLFOX4 treatment and RAS mutations in colorectal cancer. $N$ Engl J Med. 2013; 369(11):1023-1034.

26. Lenz H, Niedzwiecki D, Innocenti F, et al. 5010 CALGB/SWOG 80405: phase III trial of irinotecan/5-fu/leucovorin (folfiri) or oxaliplatin/5-fu/leucovorin (mfolfox6) with bevacizumab (bv) or cetuximab (cet) for patients (pts) with expanded ras analyses untreated metastatic adenocarcinoma of the colon or rectum (mcrc). Ann Oncol. 2014;25(suppl 4):mdu438.13.

27. Suenaga M, Schirripa M, Cao S, et al. Genetic variants of DNA repairrelated genes predict efficacy of TAS-102 in patients with refractory metastatic colorectal cancer. Ann Oncol. 2017;28(5):1015-1022.
28. Yoshino T, Shinozaki E, Yamazaki K, et al. Effect of thymidine kinase 1 expression on prognosis and treatment outcomes in refractory metastatic colorectal cancer: results from two randomized studies of TAS-102 versus a placebo. J Clin Oncol. 2017;35(4 Suppl):529.

29. Kasi PM, Kotani D, Cecchini M, et al. Chemotherapy induced neutropenia at 1-month mark is a predictor of overall survival in patients receiving TAS-102 for refractory metastatic colorectal cancer: a cohort study. BMC Cancer. 2016;16:467.

30. Temmink OH, Hoebe EK, van der Born K, Ackland SP, Fukushima M, Peters GJ. Mechanism of trifluorothymidine potentiation of oxaliplatininduced cytotoxicity to colorectal cancer cells. Br J Cancer. 2007; 96(2):231-240.

31. Temmink OH, Hoebe EK, Fukushima M, Peters GJ. Irinotecan-induced cytotoxicity to colon cancer cells in vitro is stimulated by pre-incubation with trifluorothymidine. Eur J Cancer. 2007;43(1):175-183.

32. Nukatsuka M, Nakagawa F, Takechi T. Efficacy of combination chemotherapy using a novel oral chemotherapeutic agent, TAS-102, with oxaliplatin on human colorectal and gastric cancer xenografts. Anticancer Res. 2015;35(9):4605-4615.

33. Nukatsuka M, Nakagawa F, Saito H, Sakata M, Uchida J, Takechi T. Efficacy of combination chemotherapy using a novel oral chemotherapeutic agent, TAS-102, with irinotecan hydrochloride on human colorectal and gastric cancer xenografts. Anticancer Res. 2015;35(3): 1437-1445.

34. Tsukihara H, Nakagawa F, Sakamoto K, et al. Efficacy of combination chemotherapy using a novel oral chemotherapeutic agent, TAS-102, together with bevacizumab, cetuximab, or panitumumab on human colorectal cancer xenografts. Oncol Rep. 2015;33(5):2135-2142.

35. Baba Y, Tamura T, Satoh Y, et al. Panitumumab interaction with TAS-102 leads to combinational anticancer effects via blocking of EGFR-mediated tumor response to trifluridine. Mol Oncol. 2017;11(8): 1065-1077.

36. Suzuki N, Tsukihara H, Nakagawa F, Kobunai T, Takechi T. Efficacy of trifluridine/tipiracil + anti-mouse PD-1 antibody combination on mouse colorectal cancer model and related tumor immunomodulatory effects [abstract]. In: Proceedings of the American Association for Cancer Research Annual Meeting 2017;2017 April 1-5; Washington, DC. Philadelphia (PA): AACR; Cancer Res. 2017;77(13 Suppl):Abstract nr 1071.

37. Doi T, Yoshino T, Fuse N, et al. Phase I study of TAS-102 and irinotecan combination therapy in Japanese patients with advanced colorectal cancer. Invest New Drugs. 2015;33(5):1068-1077.

38. Kuboki Y, Nishina T, Shinozaki E, et al. TAS-102 plus bevacizumab for patients with metastatic colorectal cancer refractory to standard therapies (C-TASK FORCE): an investigator-initiated, open-label, single-arm, multicentre, phase 1/2 study. Lancet Oncol. Epub 2017 July 28 .
OncoTargets and Therapy

\section{Publish your work in this journal}

OncoTargets and Therapy is an international, peer-reviewed, open access journal focusing on the pathological basis of all cancers, potential targets for therapy and treatment protocols employed to improve the management of cancer patients. The journal also focuses on the impact of management programs and new therapeutic agents and protocols on

\section{Dovepress}

patient perspectives such as quality of life, adherence and satisfaction. The manuscript management system is completely online and includes a very quick and fair peer-review system, which is all easy to use. Visit http://www.dovepress.com/testimonials.php to read real quotes from published authors. 